\begin{tabular}{|c|c|}
\hline Title & Weakly nonlinear waves generated by vibration of a spherical body \\
\hline Author(s) & Y ano, Takeru; Inoue, Y oshinori \\
\hline Citation & $\begin{array}{l}\text { The Journal of the A coustical Society of A merica, 91(1), } 69-78 \\
\text { https://doi.org/10.1121/1.402621 }\end{array}$ \\
\hline Issue Date & 1992-01 \\
\hline Doc URL & http:/hdl.handle.net/2115/44919 \\
\hline Rights & $\begin{array}{l}\text { Copyright 1992, A coustical Society of A merica. This article may be downloaded for personal use only. A ny other use } \\
\text { requires prior permission of the author and the A coustical Society of A merica. }\end{array}$ \\
\hline Type & article \\
\hline File Information & JA SA 91-1.pdf \\
\hline
\end{tabular}

Instructions for use 


\title{
Weakly nonlinear waves generated by vibration of a spherical body
}

\author{
Takeru Yano and Yoshinori Inoue \\ Department of Engineering Science, Faculty of Engineering, Hokkaido University, Sapporo 060, Japan
}

(Received 10 October 1990; accepted for publication 12 September 1991)

\begin{abstract}
The nonlinear propagation of directional spherical waves generated in an unbounded inviscid ideal gas by vibratory motions with small but finite amplitude and moderate frequency of a spherical body is considered. Starting with a regular perturbation expansion for a velocity potential in the near field, a higher-order problem is investigated in the far field up to the shock formation distance. It is thereby shown that, in the far field concerned, a well-known simple far-field equation remains valid for the radial velocity $u_{r}^{*}$ including higher-order corrections up to $O\left(\epsilon^{N} / r\right)(N<-1 / \epsilon \ln \epsilon)$, where $r$ is a nondimensional radial coordinate and $\epsilon(\ll 1)$ is the expansion parameter of the expansion. A boundary condition appropriate to the equation, which ensures the matching of a far-field solution with a near-field solution, can be determined from the near-field solution obtained by the regular perturbation procedure. As an application of the theory, the third-order problem is solved for weakly nonlinear acoustic waves radiated by a pulsating sphere. It is further shown that, for weakly nonlinear cylindrical waves with moderate frequency, a similar far-field equation becomes invalid at the third approximation in the far field up to the shock formation distance.
\end{abstract}

PACS numbers: $43.25 . \mathrm{Cb}$

\section{INTRODUCTION}

We shall consider the weakly nonlinear propagation of directional spherical waves radiated by vibratory motions of a spherical body in an inviscid ideal gas. By directional spherical waves, we mean spherically diverging waves whose amplitude can vary with angles at a distance $r^{*}$ from the center of the mean surface of the body (the mean surface is supposed to be a sphere of radius $R$ ). We shall treat the case of $\epsilon \equiv a / R \ll 1$ and $\omega \equiv R \omega^{*} / c=O(1)$, where $\epsilon$ is a normalized amplitude and $\omega$ is a normalized frequency ( $a$ : the maximum amplitude of vibrations of the body; $\omega^{*}$ : a typical angular frequency of the vibration; $c$ : the speed of sound in an undisturbed gas). ${ }^{1}$ The radiated sound field is then composed of the near and far field (see Fig. 1). In this case, an approximate solution valid in the near field, i.e., a near-field solution, can, in principle, be obtained up to ary order of $\epsilon$ by a regular perturbation expansion in powers of $\epsilon$ for a velocity potential in the near field. Starting with the regular perturbation expansion, we shall investigate a higher-order problem up to $O\left(\epsilon^{N} / r\right)$ in the far field up to the shock formation distance ( $N$ : a positive integer; $r \equiv r^{*} / R$ : a normalized distance).

The nonlinear propagation in the far field in the case of $\epsilon \ll 1$ and $\omega=O(1)$ has been well examined up to $O(\epsilon / r)::^{2-6}$ To the propagation of directional spherical waves, Kelly and Nayfeh ${ }^{2,3}$ applied the method of renormalization, which had already been used for cylindrical waves by Ginsberg. ${ }^{7}$ The problem of directional spherical waves is reconsidered in the approximation up to $O\left(\epsilon^{N} / r\right)(N \geqslant 2)$, in the present paper. Inoue and co-workers ${ }^{4-6}$ studied problems of a uniformly pulsating sphere ${ }^{4,6}$ and that of an oscillating rigid sphere, ${ }^{5}$ using a method of strained coordinates to obtain an explicit expression of an exact solution of a well-known simple far-field equation: $:^{8,9}$

$$
\begin{aligned}
& \frac{\partial w}{\partial z}-w \frac{\partial w}{\partial \varphi}=0, \\
& w=\frac{r^{*}}{R} \frac{u_{r}^{*}}{c}, z=\frac{\gamma+1}{2} \frac{R \omega^{*}}{c} \ln \frac{r^{*}}{R}, \\
& \varphi=\omega^{*}\left[t^{*}-\left(r^{*}-R\right) / c\right],
\end{aligned}
$$

where $u_{r}^{*}$ is the radial component of the fluid velocity, $\gamma$ is the ratio of specific heats, and $t^{*}$ is the time. In the approximation up to $O\left(\epsilon^{2} / r\right)$ in the far field, however, very few problems have been solved. ${ }^{4,10}$ We remark that the directivity can cause significant phenomena in the problem concerned: The acoustic streaming can be induced in the near field, ${ }^{5,10}$ and in the far field the shock formation distance depends upon the polar angle $\theta$ and the azimuthal angle $\psi^{2,3,5,10}$

In this paper, we emphasize that Eq. (1) can be derived even in the framework of the higher-order problem: Strictly speaking, Eq. (1) remains valid in the far field for $u_{r}^{*}$ including corrections up to $O\left(\epsilon^{N} / r\right)(N<-1 / \epsilon \ln \epsilon)$. In the far field the other components of the velocity, $u_{\theta}^{*}$ and $u_{\psi}^{*}$, become very small compared with $u_{r}^{*}$ so that the leading equation governing the nonlinear wave motion concerned could be reduced to a spatially one-dimensional component, i.e., Eq. (1). We shall also prescribe a boundary condition, in each order, appropriate to Eq. (1). This boundary condition ensures the matching of a far-field solution up to $O\left(\epsilon^{N} / r\right)$ with a near-field solution up to $O\left(\epsilon^{N}\right)$. Solving Eq. (1) under the appropriate boundary condition yields a far-field solution for $u_{r}^{*}$ accurate up to $O\left(\epsilon^{N} / r\right)$. The velocity potential 


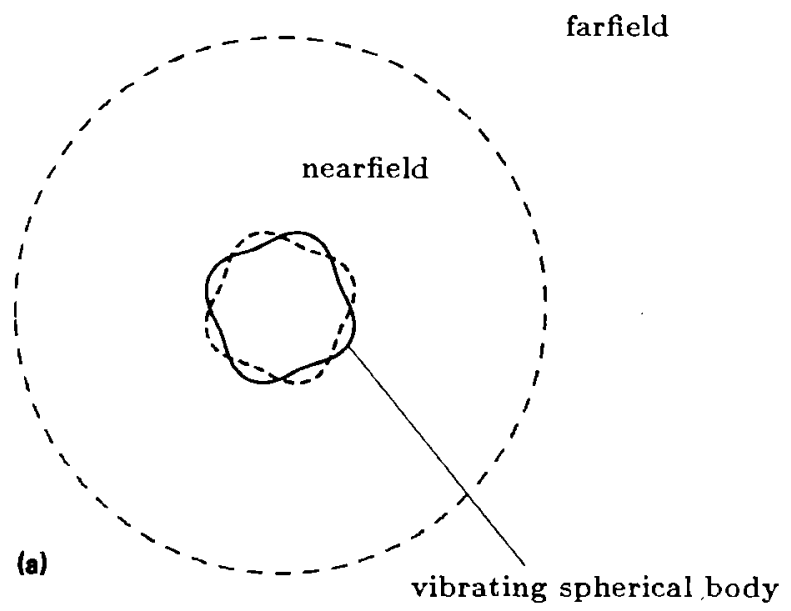

farfield

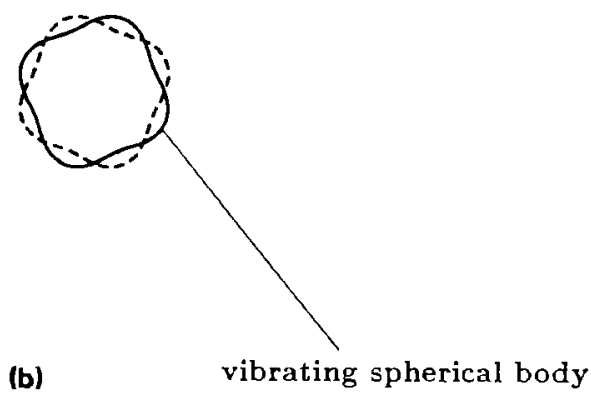

FIG. 1. The structures of the sound field radiated by a vibrating spherical body.' (a) The present case; $\epsilon \& 1$ and $\omega=O(1)$. The acoustic field is composed of the near and far field. The near field is a region of which the distances from a sound source are comparable with a typical wavelength. (b) The high-frequency case; $\epsilon \ll 1$ and $\omega \gg 1$. A typical wavelength is very small compared with the mean radius of the vibrating body. The overall acoustic field may be regarded as the far field.

valid in the far field can be given by integrating the far-field solution for $u_{r}^{*}$ with respect to $r^{*} .^{5,10}$ By differentiating the velocity potential, one can also evaluate the far-field solutions for $u_{\theta}^{*}, u_{\psi}^{*}$, and the pressure. ${ }^{5,10}$ This paper will therefore establish a systematical method to solve the higher-order problem concerned.

Naugol'nykh et al. ${ }^{8}$ and Blackstock ${ }^{9}$ studied the propagation of uniform spherical waves and derived Eq. (1) in the leading approximation, neglecting cubic terms in a basic equation for a velocity potential $\Phi^{*}$ and simplifying the resulting equation by use of the linear relation $c \partial \Phi^{*} / \partial r^{*} \sim-\partial \Phi^{*} / \partial t *$ in quadratic terms. The problems treated in Refs. 8 and 9 however pertain to a case different from the present one, i.e., the case of $\epsilon \ll 1$ and $\omega \gg 1$ (highfrequency case). In this case, the near field substantially disappears (see Fig. 1), and hence the wave propagation can, in the leading approximation, be examined by solving Eq. (1) under the boundary condition at the mean surface of the vibrating body, without considering the near field explicitly, as in Refs. 8 and 9. It may be noted that Lockwood et al. ${ }^{11}$ used the method in Ref. 9 for directional spherical waves and again derived Eq. (1) [they applied Eq. (1) to the far-field radiation of a circular piston].

Our derivation method of Eq. (1) is an extension of that by Marsh et al..$^{12}$ and by Inoue et al. ${ }^{4}$ to the higher-order problem for directional spherical waves. In the first approximation, both Marsh et al. and Inoue et al. ${ }^{4}$ derived Eq. (1) from Heaps' series solution ${ }^{13}$ with a formula that can recursively determine the coefficient in the series, i.e.,

$$
p=\frac{\gamma \epsilon}{r} \sum_{n=1}^{\infty}\left(\epsilon \frac{\gamma+1}{4} \ln r\right)^{n-1} F_{n}(\varphi),
$$

with

$$
\begin{aligned}
F_{n}(\varphi)= & \frac{1}{n-1} \frac{\partial}{\partial t} \sum_{m=1}^{n-1} F_{m}(\varphi) F_{n-m}(\varphi) \\
& (n \geqslant 2), \quad F_{1}(\varphi)=f(\varphi),
\end{aligned}
$$

where $p$ is a nondimensional pressure disturbance and $\gamma \in f$ denotes the normalized pressure fluctuation at the surface of a sphere $r=1$. Heaps has constructed the series expression (3) with (4) by collecting secular terms multiplied by $\epsilon^{n}(\ln r)^{n-1} / r$ in a regular perturbation expansion in $\epsilon$ for uniform spherical waves in the high-frequency case. His solution is accurate up to $O(\epsilon / r)$ in the far field, i.e., the firstorder far-field solution. The second-order version of formula (4) has been given by Inoue et al. ${ }^{4}$ who have retained the secular terms multiplied by $\epsilon^{n}(\ln r)^{n-2} / r$.

In Sec. I we shall formulate the problem mathematically. In Sec. II, the regular perturbation expansion in $\epsilon$ is applied to a velocity potential in the near field. We shall extend the Heaps' result, Eq. (3) with Eq. (4), up to $O\left(\epsilon^{N} / r\right)$ for directional spherical waves, by keeping all terms multiplied by $\epsilon^{n}(\ln r)^{n-m} / r(n \geqslant m \geqslant 1)$ but neglecting terms multiplied by $1 / r^{n}(n \geqslant 2)$. As a result, in the far field concerned, $w$ is expressed in the form of an asymptotic expansion in $\epsilon$, and formulas by Heaps and by Inoue et al. ${ }^{4}$ are extended. A limitation on $N$ is examined in this section. In Sec. III, we shall derive Eq. (1) from the formula obtained in Sec. II and prescribe a boundary condition appropriate to Eq. (1). In Sec. IV, we shall apply the present result to the third-order problem for uniform spherical waves radiated by a pulsating sphere (see also Ref. 4). An application for directional spherical waves can be found in Ref. 10. Section $V$ is devoted to a brief discussion of a far-field equation for cylindrical waves. We shall demonstrate that the far-field equation, ${ }^{9,14}$ which has the same form as Eq. (1) with the different definitions for $w$ and $z$, becomes invalid at the third approximation in the far field up to the distance of shock formation.

\section{FORMULATION OF THE PROBLEM}

We shall consider the propagation of weakly nonlinear acoustic waves produced in an unbounded inviscid ideal gas by vibratory motions of a spherical body. The mean surface of the body is supposed as being a sphere of radius $R$ centered at the origin in the spherical coordinates $\left(r^{*}, \theta, \psi\right)$, where $r^{*}$ is the distance from the origin, $\theta$ is the polar angle, and $\psi$ is the azimuthal angle. It is assumed that the wave motion concerned can be described in terms of a velocity potential 
$\Phi^{*}\left(r^{*}, \theta, \psi, t^{*}\right)$, where $t^{*}$ is the time. The following dimensionless quantities are introduced:

$$
\Phi=\frac{\Phi^{*}}{c R}, \quad t=\frac{t^{*} c}{R}, \quad r=\frac{r^{*}}{R},
$$

where $c$ denotes the speed of sound in an undisturbed gas. The nondimensional velocity components are given by

$$
u_{r}=\frac{\partial \Phi}{\partial r}, \quad u_{\theta}=\frac{1}{r} \frac{\partial \Phi}{\partial \theta}, \quad u_{\psi}=\frac{1}{r \sin \theta} \frac{\partial \Phi}{\partial \psi} .
$$

The momentum equations, the equation of mass continuity, and the isentropic relation are combined to yield the equation governing the nondimensional velocity potential $\Phi:$

$$
\begin{aligned}
\square \Phi= & \frac{\partial}{\partial t}(\nabla \Phi)^{2}+(\gamma-1) \Delta \Phi \frac{\partial \Phi}{\partial t} \\
& +\frac{\gamma-1}{2} \Delta \Phi(\nabla \Phi)^{2}+\frac{1}{2} \nabla \Phi \cdot \nabla(\nabla \Phi)^{2},
\end{aligned}
$$

where $\gamma$ is the ratio of specific heats, $\nabla$ is the nondimensional spatial gradient, $\Delta$ is the Laplacian, and $\square$ is the D'Alembertian operator defined as ${ }^{15}$

$$
\begin{aligned}
\square \equiv \Delta-\frac{\partial^{2}}{\partial t^{2}} \\
\equiv \frac{1}{r^{2}}\left[\frac{\partial}{\partial r}\left(r^{2} \frac{\partial}{\partial r}\right)+\frac{1}{\sin \theta} \frac{\partial}{\partial \theta}\left(\sin \theta \frac{\partial}{\partial \theta}\right)\right. \\
\left.\quad+\frac{1}{\sin ^{2} \theta} \frac{\partial^{2}}{\partial \psi^{2}}\right]-\frac{\partial^{2}}{\partial t^{2}} .
\end{aligned}
$$

The boundary condition on the surface of the body is

$$
\begin{aligned}
\frac{\partial \Phi}{\partial r}= & \frac{\partial s}{\partial t}+\frac{1}{r^{2}} \frac{\partial \Phi}{\partial \theta} \frac{\partial s}{\partial \theta} \\
& +\frac{1}{r^{2} \sin ^{2} \theta} \frac{\partial \Phi}{\partial \psi} \frac{\partial s}{\partial \psi} \text { at } r=s(\theta, \psi, t ; \epsilon) .
\end{aligned}
$$

Here, the instantaneous surface of the spherical body is supposed to be prescribed by the relation $r=s(\theta, \psi, l ; \epsilon)$, where $\epsilon \equiv a / R$ is a normalized amplitude of the body's vibratory motion ( $a$ is the maximum amplitude of the motion). Note that $\epsilon$ equals the maximum value of $|s(\theta, \psi, t ; \epsilon)-1|$. What follows is concerned with the wave phenomena characterized by the condition

$$
\epsilon \equiv a / R \ll 1 \text { and } \omega \equiv R \omega^{*} / c=O(1),
$$

where $\omega^{*}$ signifies a typical angular frequency of the sound source and $\omega$ is a normalized angular frequency. The condition (10) means that a typical wavelength of the radiated sound, $2 \pi c / \omega^{*}$, is comparable to the mean radius $R$ and that an acoustic Mach number $M \equiv a \omega^{*} / c=\epsilon \omega$ is sufficiently small. The present analysis requires no further constraint for motions of the body. The radiation condition should be imposed on the velocity potential at infinity.

\section{APPROXIMATE SOLUTION BY A REGULAR PERTURBATION EXPANSION}

We shall try to determine the solution of the problem by a regular perturbation expansion in powers of $\epsilon$ :

$$
\Phi(r, \theta, \psi, t)=\sum_{n=1}^{\infty} \epsilon^{n} \Phi_{n}(r, \theta, \psi, t) .
$$

It then follows from Eqs. (6) and (11) that

$$
u_{r}(r, \theta, \psi, t)=\sum_{n=1}^{\infty} \epsilon^{n} \frac{\partial \Phi_{n}}{\partial r}(r, \theta, \psi, t) .
$$

Substituting expansion (11) into Eq. (7) and equating coefficients of like powers of $\epsilon$ gives a successive set of equations:

$O(\epsilon): \square \Phi_{1}=0$,

$$
\begin{aligned}
O\left(\epsilon^{2}\right): \square \Phi_{2}= & \frac{\partial}{\partial t}\left[\frac{\gamma-1}{2}\left(\frac{\partial \Phi_{1}}{\partial t}\right)^{2}+\left(\frac{\partial \Phi_{1}}{\partial r}\right)^{2}\right. \\
& \left.+\left(\frac{1}{r} \frac{\partial \Phi_{1}}{\partial \theta}\right)^{2}+\left(\frac{1}{r \sin \theta} \frac{\partial \Phi_{1}}{\partial \psi}\right)^{2}\right]
\end{aligned}
$$

$$
\begin{aligned}
O\left(\epsilon^{n}\right): \square \Phi_{n}= & \frac{\partial}{\partial t} \sum_{m=1}^{n-1}\left(\frac{\gamma-1}{2} \frac{\partial \Phi_{m}}{\partial t} \frac{\partial \Phi_{n} m}{\partial t}\right. \\
& +\frac{\partial \Phi_{m}}{\partial r} \frac{\partial \Phi_{n-m}}{\partial r}+\frac{1}{r^{2}} \frac{\partial \Phi_{m}}{\partial \theta} \frac{\partial \Phi_{n-m}}{\partial \theta} \\
& \left.+\frac{1}{r^{2} \sin ^{2} \theta} \frac{\partial \Phi_{m}}{\partial \psi} \frac{\partial \Phi_{n-m}}{\partial \psi}\right)+\mathrm{CQT}
\end{aligned}
$$

(13n)

where CQT on the right-hand side of Eq. (13n) signifies terms proportional to, at least, $1 / r^{3}$, since $\Phi_{m}$ has a geometrical decaying factor $1 / r$. CQT can therefore be ignored in seeking an asymptotic solution at large $r$. The function $s(\theta, \psi, t ; \epsilon)$, which prescribes the instantaneous surface of the body, may, in general, be expanded in the form

$$
s(\theta, \psi, t ; \epsilon)=1+\sum_{n=1}^{\infty} \epsilon^{n} S_{n}(\theta, \psi, t) .
$$

Substituting expansions (11) and (14) into the boundary condition (9) and equating coefficients of like powers of $\epsilon$ gives the boundary conditions

$$
\begin{aligned}
O(\epsilon):\left.\frac{\partial \Phi_{1}}{\partial r}\right|_{r=1}= & \frac{\partial s_{1}}{\partial t}, \\
O\left(\epsilon^{2}\right):\left.\frac{\partial \Phi_{2}}{\partial r}\right|_{r=1}= & \frac{\partial s_{2}}{\partial t}-\left.s_{1} \frac{\partial^{2} \Phi_{1}}{\partial r^{2}}\right|_{r=1}+\left.\frac{\partial s_{1}}{\partial \theta} \frac{\partial \Phi_{1}}{\partial \theta}\right|_{r=1} \\
& +\left.\frac{1}{\sin ^{2} \theta} \frac{\partial s_{1}}{\partial \psi} \frac{\partial \Phi_{1}}{\partial \psi}\right|_{r=1},
\end{aligned}
$$

and so forth.

The system (13) can, in principle, be solved successively in conjunction with the boundary conditions (15). However, since our purpose is to investigate the acoustic behavior in the far field, all that is required are the asymptotic forms of the $\Phi_{n}$ 's as $r \rightarrow \infty$. As is readily verified, $\Phi_{1}$ satisfying the radiation condition has the asymptotic form

$$
\Phi_{1}=(1 / r) f_{1}^{(1)}(\varphi, \theta, \psi)+O\left(1 / r^{2}\right) \quad \text { as } r \rightarrow \infty \text {. }
$$

The functional form of $f_{1}^{(1)}(\varphi, \theta, \psi)$ may immediately be determined provided that the function $s_{1}$ is explicitly given. 
According to Refs. 13 and 4, we assume that at large distances from the body,

$\Phi_{m}=\frac{1}{r} \sum_{k=1}^{m}\left(\frac{\gamma+1}{4} \ln r\right)^{m-k} f_{m}^{(k)}(\varphi, \theta, \psi) \quad(m=2,3, \ldots)$,

where the functions $f_{m}^{(k)}$ of $O(1)$ are at present undetermined. Without loss of generality, one can let $f_{m}^{(k)} \equiv 0$ for $m<k$. Substituting Eq. (17) into both sides of Eq. (13n) and equating the coefficients of like powers of $\ln r$ yields

$$
\begin{aligned}
F_{n}^{(k)}= & \frac{1}{n-k} \frac{\partial}{\partial t} \sum_{m=1}^{n-1} \sum_{j=1}^{k} F_{m}^{(j)} F_{n-m}^{(k+1-j)} \\
& (n=k+1, k+2, \ldots ; k=1,2, \ldots),
\end{aligned}
$$

where $F_{n}^{(k)}(\varphi, \theta, \psi) \equiv-\partial f_{n}^{(k)} / \partial t$. Once the $F_{k}^{(k)}$ s s are explicitly found for all $k$ up to $N$ ( $N$ : a positive integer), one can recursively determine the $F_{n}^{(k)}$ 's, for any $n$ but for all $k$ up to $N$, by using formula (18) and the $F_{k}^{(k)}$ 's. A limitation on $N$ will be examined later. It should be emphasized, however, that to obtain the explicit form of the function $F_{k}^{(k)}$, the boundary value problem for $\Phi_{k}$ needs to be solved exactly. Heaps' formula (4) is the simplest version of formula (18), i.e., that in the case of $k=1$, which implies the first approximation at large $r$. Inoue et al. ${ }^{4}$ have recently extended Heaps' formula (4) to the case of $k=2$ corresponding to the second approximation at large $r$. Formula (18) is an extension of them to higher-order problems for directional spherical waves.

Consequently, an asymptotic form at large $r$ of the $N$ thorder near-field solution for $u_{r}$ can be expressed, from Eqs. (12) and (17), as

$$
r u_{r}=\sum_{n=1}^{N} \epsilon^{n} \sum_{k=1}^{N}\left(\frac{\gamma+1}{4} \ln r\right)^{n-k} F_{n}^{(k)},
$$

where one may recall that $F_{n}^{(k)} \equiv 0$ for $n<k$. However, at very large $r$ such that $\epsilon \ln r \sim 1$, where a shock wave can be formed, ${ }^{4}$ the contribution of the secular terms neglected in Eq. (19), namely, $\epsilon^{n}(\ln r)^{n-k}(n>N)$, becomes comparable to that of $\epsilon^{n}(\ln r)^{n-k}(n \leqslant N)$. A representation appropriate for such a region may therefore be

$$
r u_{r}=\sum_{n=1}^{\infty} \epsilon^{n} \sum_{k=1}^{N}\left(\frac{\gamma+1}{4} \ln r\right)^{n-k} F_{n}^{(k)} .
$$

We here note that all the $F_{n}^{(k)}$ 's appearing in Eq. (20) can recursively be determined by means of formula (18) if only the $F_{k}^{(k)}$ 's are known up to $k=N$.

Introducing a new coordinate $Z$ defined as

$$
Z \equiv \epsilon z / 2 \omega \equiv \epsilon[(\gamma+1) / 4] \ln r,
$$

and interchanging the order of sums in Eq. (20) leads to

$$
w(Z, \varphi, \theta, \psi) \equiv r u_{r}=\sum_{k=1}^{N} \epsilon^{k} w_{k}(Z, \varphi, \theta, \psi),
$$

where the function $w_{k}$ is defined as

$w_{k}(Z, \varphi, \theta, \psi) \equiv \sum_{n=1}^{\infty} Z^{n-k} F_{n}^{(k)}(\varphi, \theta, \psi) \quad(k=1,2, \ldots, N)$.

Equation (22) may be an asymptotic expansion for $r u_{r}$ in the far field. In particular, the first term in the expansion (22), i.e., $\epsilon w_{1}$, can be transformed into the Fubini solution if the body's vibratory motion is sinusoidal in time in $O(\epsilon){ }^{13,4} \mathrm{An}$ expression given by Heaps for the first-order far-field pressure, Eq. (3), is the counterpart of $\gamma \epsilon w_{1} / r$.

At this point we should remark that an inequality

$$
\epsilon^{N} / r \gg \epsilon / r^{2} \text {, }
$$

should hold in the far field up to the distance of shock formation since the contribution of the terms multiplied by $1 / r^{n}(n \geqslant 2)$ to $u_{r}$ is neglected throughout the analysis in this section. The inequality (24) may be satisfied for moderately large $N$ less than $-1 / \epsilon \ln \epsilon$ in the region, because the shock formation distance $r_{s} \sim \exp (1 / \epsilon){ }^{4}$

Making use of Heaps' formula (4), Marsh et al. ${ }^{12}$ and Inoue et al. $^{4}$ have derived Eq. (1) as an equation which governs $\epsilon w_{1}$. In the next section, by developing their approach, we shall derive Eq. (1) from formula (18) as an equation that governs $w$ given by Eq. (22). It may be noted that, if $F_{k}^{(k)}$ is an analytic function of $\varphi$, the right-hand side of Eq. (23) is a convergent series (Appendix D).

\section{DERIVATION OF A FAR-FIELD EQUATION}

The process of derivation of the far-field equation (1) can be divided into two steps. First, we will determine equations for the $w_{k}$ 's defined by Eq. (23). Multiplying formula (18) by $Z^{n-k}$ gives

$$
\begin{aligned}
Z^{n-k} F_{n}^{(k)}= & \frac{1}{n-k} Z^{n-k} \frac{\partial}{\partial t} \sum_{m=1}^{n-1} \sum_{j=1}^{k} F_{m}^{(j)} F_{n-m}^{(k+1-j)} \\
& (n=k+1, k+2, \ldots ; k=1,2, \ldots)
\end{aligned}
$$

It then follows that

$$
\begin{aligned}
\sum_{n=k+1}^{\infty} Z^{n-k} F_{n}^{(k)}= & \frac{\partial}{\partial t} \int_{0}^{Z} \sum_{n=k+1}^{\infty} \sum_{m=1}^{n-1} \sum_{j=1}^{k} Z^{n-k-1} \\
& \times F_{m}^{(j)} F_{n-m}^{(k+1-j)} d Z \\
& (k=1,2, \ldots) .
\end{aligned}
$$

Adding $F_{k}^{(k)}$ to both sides of Eq. (26) and recalling that $F_{n}^{(k)} \equiv 0$ for $n<k$, one can obtain

$$
\begin{aligned}
\sum_{n=1}^{\infty} Z^{n-k} F_{n}^{(k)}= & \frac{\epsilon}{2} \frac{\partial}{\partial \varphi} \int_{0}^{z} \sum_{n=2}^{\infty} \sum_{m=1}^{n-1} \sum_{j=1}^{k} Z^{n-k-1} F_{m}^{(j)} \\
& \times F_{n-m}^{(k+1-j)} d z+F_{k}^{(k)} \\
& (k=1,2, \ldots)
\end{aligned}
$$

where the relations $\varphi=\omega(t-r+1)$ and $Z=\epsilon z / 2 \omega$ have been used. Substituting Eq. (23) into Eq. (27) yields the equations governing the $w_{k}$ 's:

$$
w_{k}=\frac{\epsilon}{2} \frac{\partial}{\partial \varphi} \int_{0}^{z} \sum_{j=1}^{k} w_{j} w_{k+1-j} d z+F_{k}^{(k)} \quad(k=1,2, \ldots)
$$

(see Refs. 4 and 12). In particular, for $k=1$, it may immediately be recognized from Eq. (28) that $\epsilon w_{1}$ is the solution of Eq. (1) satisfying the boundary condition

$$
\epsilon w_{1}(0, \varphi, \theta, \psi)=\epsilon F_{1}^{(1)}(\varphi, \theta, \psi) \text { at } z=0 \text {. }
$$

Next we will construct an equation that governs $w$ given by Eq. (22). From Eq. (28) one can readily get 


$$
\begin{aligned}
\sum_{k=1}^{N} \epsilon^{k} w_{k}= & \frac{1}{2} \frac{\partial}{\partial \varphi} \int_{0}^{z}\left[\left(\sum_{k=1}^{N} \epsilon^{k} w_{k}\right)^{2}\right. \\
& \left.-\sum_{\substack{N+2<p+q \\
2<p, q<N}} \epsilon^{p+q} w_{p} w_{q}\right] d z+\sum_{k=1}^{N} \epsilon^{k} F_{k}^{(k)}
\end{aligned}
$$

Equation (30) can then be rewritten into

$w=\frac{1}{2} \frac{\partial}{\partial \varphi} \int_{0}^{z}\left[w^{2}+O\left(\epsilon^{N+2}\right)\right] d z+\sum_{k=1}^{N} \epsilon^{k} F_{k}^{(k)}$.

Differentiating Eq. (31) with respect to 2, one can obtain the far-field equation accompanied with permissible error terms:

$$
\frac{\partial w}{\partial z}-w \frac{\partial w}{\partial \varphi}+O\left(\epsilon^{N+2}\right)=0 .
$$

That is, the nonlinear propagation of spherical acoustic waves in the far field is, in the $N$ th-order problem, described by Eq. (1) and the boundary condition

$$
w(0, \varphi, \theta, \psi)=\sum_{k=1}^{N} \epsilon^{k} F_{k}^{(k)}(\varphi, \theta, \psi) \quad \text { at } z=0 .
$$

The right-hand side of Eq. (33) denotes the value at $z=0$ of the right-hand side of Eq. (19), namely, the asymptotic form at large $r$ of the $N$ th-order near-field solution for $u_{r}$. The $N$ th-order far-field solution for $u_{r}$ is immediately given from $w$ which is the solution of Eq. (1) satisfying the boundary condition (33). It is evident that this $N$ th-order far-field solution matches with the near-field solution up to $O\left(\epsilon^{N}\right)$, which can, in principle, be constructed by the regular perturbation procedure in Sec. II. The far-field solutions for the other components of the velocity, $u_{\theta}$ and $u_{\psi}$, and also for the pressure can readily be calculated from $u_{r}$ (see Refs. 5 and 10). Note that Appendix $D$ gives a mathematical justification for the present method of derivation of the far-field equation.

\section{APPLICATION: HARMONICALLY PULSATING SPHERE}

\section{A. The third-order solution}

As an application, we shall examine the propagation of weakly nonlinear acoustic waves emitted from a sphere which pulsates harmonically with amplitude $a$ and frequency $\omega^{*}$. The problem is one of fundamental importance in nonlinear acoustics and therefore not only the first- but the second-order solution has already been obtained and has been analyzed in detail. ${ }^{4}$ Accordingly, we here concentrate on the third-order problem. Another application can be found in Ref. 10, where the second-order problem is solved for the nonlinear propagation of directional spherical waves radiated by an oscillating rigid sphere.

The function $s(t ; \epsilon)$ which prescribes the instantaneous radius of a harmonically pulsating sphere has, e.g., the form ${ }^{4}$

$$
s=1+\epsilon \cos \omega t,
$$

where the condition (10) in Sec. I should be satisfied. The boundary value problem for $\Phi_{3}$ can be formulated as follows:

$$
\begin{aligned}
& \square \Phi_{3}= A^{3} \omega^{7}\left[\left(\frac{3}{2 r^{6} \omega^{6}}+\frac{a_{1}}{r^{4} \omega^{4}}+\frac{a_{2}}{r^{3} \omega^{3}}+\frac{a_{3}}{r^{2} \omega^{2}}\right) \sin 3 \varphi\right. \\
&+\left(-\frac{1}{2 r^{7} \omega^{7}}+\frac{5 \gamma+33}{8 r^{5} \omega^{5}}+\frac{a_{4}}{r^{4} \omega^{4}}+\frac{a_{5}}{r^{3} \omega^{3}}+\frac{a_{6}}{r^{2} \omega^{2}}\right) \\
& \times \cos 3 \varphi+\left(\frac{3}{2 r^{6} \omega^{6}}+\frac{a_{7}}{r^{4} \omega^{4}}+\frac{a_{8}}{r^{3} \omega^{3}}+\frac{a_{9}}{r^{2} \omega^{2}}\right) \sin \varphi \\
&+\left(-\frac{3}{2 r^{7} \omega^{7}}-\frac{\gamma-3}{8 r^{5} \omega^{5}}+\frac{a_{10}}{r^{4} \omega^{4}}+\frac{a_{11}}{r^{3} \omega^{3}}+\frac{a_{12}}{r^{2} \omega^{2}}\right) \\
&\left.\frac{\partial \Phi_{3}}{\partial r}\right|_{r=1}=A^{3}\left[b_{1} \sin (3 \omega t+3 \delta)+b_{2} \cos (3 \omega t+3 \delta)\right. \\
&\left.\quad+b_{3} \sin (\omega t+\delta)+b_{4} \cos (\omega t+\delta)\right],
\end{aligned}
$$

where $A \equiv \omega / \sqrt{1+\omega^{2}}$ and $\delta \equiv \arctan (1 / \omega)$; the $a_{i}$ 's $(1 \leqslant i \leqslant 12)$ are functions of $r$ and the $b_{i}$ 's are constants dependent upon $\gamma$ and $\omega$, whose explicit forms are presented in Appendix B. Note that, in this section, the definition of a phase function $\varphi$ is ${ }^{4}$

$$
\varphi=\omega(t-r+1)+\delta .
$$

The exact solution for $\Phi_{3}$ can be obtained as

$$
\begin{aligned}
\Phi_{3}= & A^{3} \omega^{5}\left[\left(\frac{3}{40 r^{4} \omega^{4}}+\frac{c_{1}}{r^{2} \omega^{2}}+\frac{c_{2}}{r \omega}\right) \sin 3 \varphi\right. \\
& +\left(-\frac{1}{40 r^{5} \omega^{5}}+\frac{c_{3}}{r^{3} \omega^{3}}+\frac{c_{4}}{r^{2} \omega^{2}}+\frac{c_{5}}{r \omega}\right) \cos 3 \varphi \\
& +\left(\frac{3}{40 r^{4} \omega^{4}}+\frac{c_{6}}{r^{2} \omega^{2}}+\frac{c_{7}}{r \omega}\right) \sin \varphi \\
& \left.+\left(-\frac{3}{40 r^{5} \omega^{5}}+\frac{c_{8}}{r^{3} \omega^{3}}+\frac{c_{9}}{r^{2} \omega^{2}}+\frac{c_{10}}{r \omega}\right) \cos \varphi\right],
\end{aligned}
$$

where the $c_{i}$ 's $(1 \leqslant i \leqslant 10)$ are functions of $r$ defined in Appendix C. $\Phi_{3}$ consists of the fundamental and third harmonics. We here remark that $\Phi_{2}$ has not only the second harmonics but a term independent of the time $t$, which implies the existence of a steady volume inflow. ${ }^{4}$ The explicit forms of $\Phi_{1}$ and $\Phi_{2}$ are presented in Appendix $A$ (see Ref. 4 for details).

Now the functions $F_{1}^{(1)}, F_{2}^{(2)}$, and $F_{3}^{(3)}$ can explicitly be written, from Eqs. (38), (A1), and (A2) in Appendix A, as $F_{1}^{(1)}=-A \omega \sin \varphi, \quad F_{2}^{(2)}=A^{2} \omega^{2} B \sin (2 \varphi+\alpha)$,

$F_{3}^{(3)}=-A^{3} \omega^{3}\left[C_{1} \sin \left(3 \varphi+\beta_{1}\right)+C_{2} \sin \left(\varphi+\beta_{2}\right)\right]$,

where $B, C_{1}, C_{2}, \alpha, \beta_{1}$, and $\beta_{2}$ are constants dependent upon $\gamma$ and $\omega$, which are also defined in Appendix C. From Eqs. (33) and (39), the boundary condition for the third-order far-field problem becomes

$$
\begin{aligned}
w= & -\epsilon A \omega \sin \varphi+(\epsilon A \omega)^{2} B \sin (2 \varphi+\alpha) \\
& -(\epsilon A \omega)^{3}\left[C_{1} \sin \left(3 \varphi+\beta_{1}\right)+C_{2} \sin \left(\varphi+\beta_{2}\right)\right] \\
& \text { at } z=0 .
\end{aligned}
$$

The solution of Eq. (1) satisfying the boundary condition 
(40) can then be obtained, in implicit form, as

$$
\begin{aligned}
w=r u_{r}= & -\epsilon A \omega \sin \hat{\varphi}+(\epsilon A \omega)^{2} B \sin (2 \hat{\varphi}+\alpha) \\
& -(\epsilon A \omega)^{3}\left[C_{1} \sin \left(3 \hat{\varphi}+\beta_{1}\right)+C_{2} \sin \left(\hat{\varphi}+\beta_{2}\right)\right],
\end{aligned}
$$

$\hat{\varphi} \equiv \varphi+z w$.

We here have used the fact that $\varphi+z w=\hat{\varphi}$ is a characteristic curve of Eq. (1). Solving the equation $\hat{\varphi}=\varphi+z w(\hat{\varphi})$ with the help of the functional form of $w(\hat{\varphi})$, we have $\hat{\varphi}$ as a function of $\varphi$ and $z$, and hence we can determine $w$ as a function of $\varphi$ and $z$. In Ref. 4, the far-field solution to the second approximation has been constructed by the method of renormalization; the result is, up to $O\left(\epsilon^{2} / r\right)$, equivalent to Eq. (41).

In Fig. 2, a wave profile of the third-order solution is depicted and compared with those of the first- and the second-order solutions. Clearly, the third-order correction on the waveform distortion is very small.

\section{B. Shock formation distance}

Beyond the point where

$$
\frac{\partial u_{r}}{\partial r}=-\infty,
$$

the radial velocity given by Eq. (41) becomes multiple valued. Clearly, the determination of the nearest location for a shock front amounts to finding the lowest value $z_{s}$ and the corresponding value of a phase function $\varphi_{s}$ satisfying

$$
\frac{\partial w}{\partial \varphi}=\infty
$$

From Eqs. (41) and (43), $z_{s}$ can be determined as

$$
\begin{aligned}
z_{s}=\{ & -\epsilon A \omega \cos \hat{\varphi}_{s}+2(\epsilon A \omega)^{2} B \cos \left(2 \hat{\varphi}_{s}+\alpha\right) \\
& -(\epsilon A \omega)^{3}\left[3 C_{1} \cos \left(3 \hat{\varphi}_{s}+\beta_{1}\right)\right. \\
& \left.\left.+C_{2} \cos \left(\hat{\varphi}_{s}+\beta_{2}\right)\right]\right\}^{-1}
\end{aligned}
$$

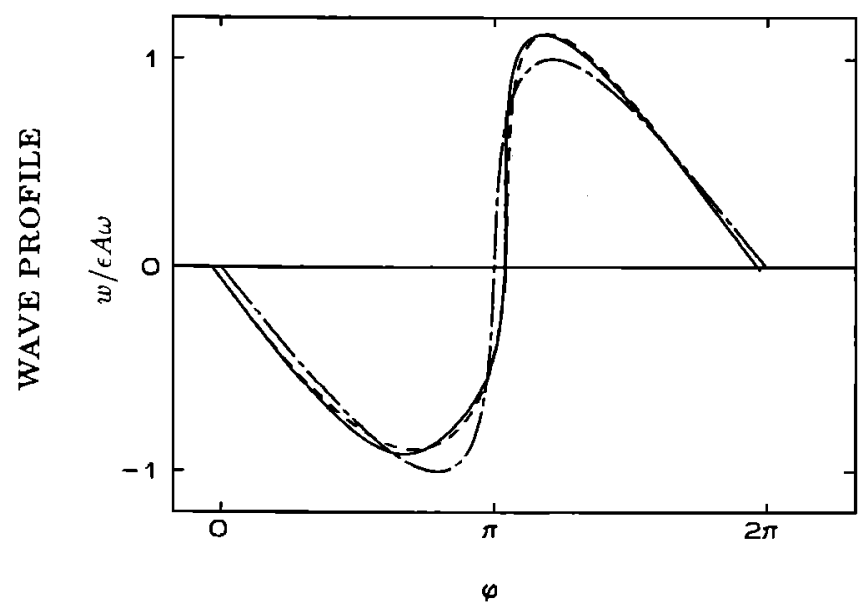

FIG. 2. Wave profiles at the distance of shock formation $z_{\mathrm{s}}$ in the case of $\epsilon=0.035, \omega=2.5$, and $\gamma=1.4 ; z_{s}=11.2$ in this case. The solid curve denotes the third-order solution, Eq. (41). The broken and dash-dotted curves are the second- and the first-order solution, respectively.
Here, $\hat{\varphi}_{s}$ should be determined as the value of $\hat{\varphi}$ that makes $d w(\hat{\varphi}) / d \hat{\varphi}$ maximum. The distance of shock formation $r_{s}$ can thus be given by

$$
r_{s}=\exp \left[2 z_{s} /(\gamma+1) \omega\right],
$$

where the relation $z=[(\gamma+1) / 2] \omega \ln r$ has been used.

Figure 3 shows the shock formation distance for varying $\epsilon$. It appears from Fig. 3(a) and (b) that, for very small $\epsilon<0.01$, the first-order solution has a tendency to give smaller values for the distance of shock formation than the higher-order ones.

\section{COMMENT ON CYLINDRICAL WAVES}

In this section, the present theory is applied to cylindrical waves radiated by an infinite circular cylinder that vibrates uniformly in the axial direction. The problem of the propagation of cylindrical waves concerned can be formulated in the same manner as in Sec. I. We here again confine ourselves to the case of $\epsilon \ll 1$ and $\omega=O(1)$ [the condition (10) ]. The method of analysis employed in Secs. II and III is available, accordingly. Consequently, a far-field equation of

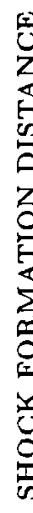

(a)

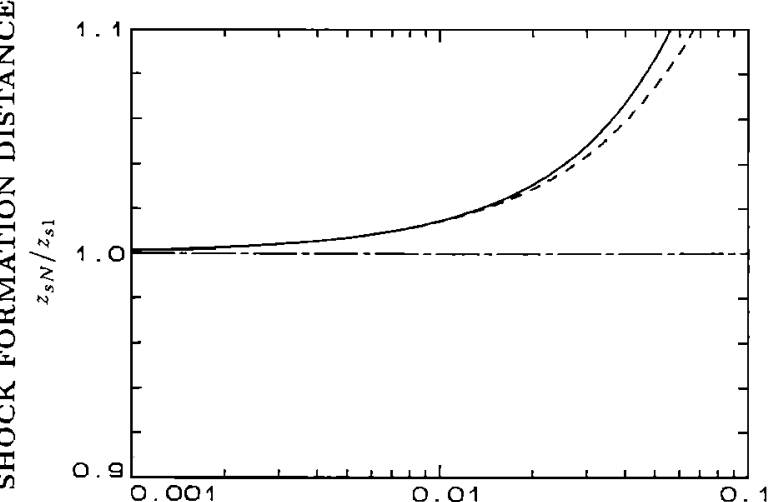

$\varepsilon$

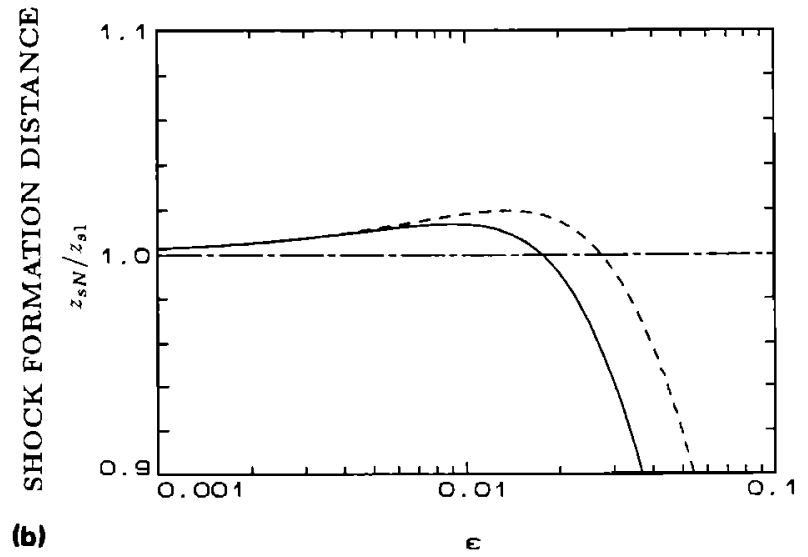

FIG. 3. The normalized shock formation distance $z_{s N} / z_{s 1}$ is depicted, where $\boldsymbol{z}_{s N}$ denotes the shock formation distance in the $N$ th-order solution $(N=1,2,3)$ [ see Eq. (44) and also Ref. 4]. The solid curve and the broken curve denote $z_{s 3} / z_{s 1}$ and $z_{s 2} / z_{s 1}$, respectively. The dash-dotted line shows $z_{s 1} / z_{s 1}=1$. (a) $\omega=0.8, \gamma=1.4$. (b) $\omega=2.5, \gamma=1.4$. 
the same form as Eq. (1) is obtained, which is identical to the equation previously derived in the case of high frequency ( $\epsilon \lll 1$ and $\omega \gg 1$ ) by Naugol'nykh et al. ${ }^{14}$ (in the inviscid limit) and by Blackstock. ${ }^{9}$ We will, however, demonstrate that the far-field equation becomes invalid at the third approximation in the far field up to the shock formation distance. It should be noted that Ginsberg ${ }^{7}$ has constructed the first-order far-field solution by the method of renormalization; the solutions for $u_{r}$ and for the pressure $p$ can consistently be included within the following analysis. ${ }^{16}$

For cylindrical waves, the asymptotic form of $\Phi_{1}$ that satisfies the radiation condition has the form ${ }^{7}$

$$
\Phi_{1}=(1 / \sqrt{r}) f_{1}^{(1)}(\varphi, \theta)+O(1 / r \sqrt{r}) \text { as } r \rightarrow \infty,
$$

where $\theta$ is the polar angle. Accordingly, instead of Eq. (17), we assume that at large distances from the cylinder,

$$
\begin{aligned}
\Phi_{m}= & \frac{1}{\sqrt{r}} \sum_{k=1}^{m}\left[\frac{\gamma+1}{2}(\sqrt{r}-1)\right]^{m-k} f_{m}^{(k)}(\varphi, \theta) \\
& (m=2,3, \ldots) .
\end{aligned}
$$

Calculations similar to those in Sec. II give a recursion formula of the same form as formula (18). An asymptotic expansion for the far-field solution, which is the counterpart of Eq. (22) with Eq. (23), can be expressed as

$$
w \equiv \sqrt{r} u_{r}=\sum_{k=1}^{N} \epsilon^{k} w_{k},
$$

with

$w_{k}(Z, \varphi, \theta) \equiv \sum_{n=1}^{\infty} Z^{n-k} F_{n}^{(k)}(\varphi, \theta) \quad(k=1,2, \ldots, N)$,

where $F_{n}^{(k)} \equiv-\partial f_{n}^{(k)} / \partial t$, and $Z$ is, in this problem, defined as

$$
Z \equiv \epsilon z / 2 \omega \equiv \epsilon[(\gamma+1) / 2](\sqrt{r}-1) .
$$

That is, we have kept all the secular terms in proportion to $\epsilon^{n}(\sqrt{r}-1)^{n-k} / \sqrt{r}(n>k>1)$ but have neglected the terms multiplied by $1 / \sqrt{r^{n}}(n \geqslant 2)$. Now, the process of derivation of a far-field equation in Sec. III can be applied in entirety, and hence one can derive the same equation as Eq. (32) with the different definitions for $w$ and $z$ (cf. Refs. 9 and 14) and obtain the same boundary condition as Eq. (33).

However, even at the shock formation distance $r_{s}$, the contribution of the neglected terms of $O\left(\epsilon / r_{s} \sqrt{r_{s}}\right)$ and $O\left(\epsilon^{2} / r_{s}\right)$ to $u_{r}$ is comparable to that of the terms of $O\left(\epsilon^{3} / \sqrt{r_{s}}\right)$, since $r_{s} \sim 1 / \epsilon^{2}$ for cylindrical waves concerned. ${ }^{7}$ Therefore, an integer $N$ must be less than three in the righthand side of Eq. (48). This means that in order to examine higher-order nonlinear effects of $O\left(\epsilon^{N} / \sqrt{r}\right)(N \geqslant 3)$ in the far field, one requires not the simple approximate equation (1) but the complicated exact equation (7) or other new farfield equations.

\section{ACKNOWLEDGMENT}

The authors would like to thank the referee for his valuable comments and criticisms.

\section{APPENDIX A: EXACT EXPRESSIONS FOR $\Phi_{1}$ AND $\Phi_{2}$}

The problem taken up as an application in Sec. IV has already been solved by Inoue $e t a l^{4}$ up to the second order. We here only present the results in the near field (see Ref. 4 for details):

$$
\Phi_{1}=-(A / r) \cos \varphi, \quad \varphi=\omega(t-r+1)+\delta,
$$

where $A=\omega / \sqrt{1+\omega^{2}}$ and $\delta=\arctan (1 / \omega)$;

$$
\begin{aligned}
\Phi_{2}= & \frac{A^{2} \omega}{r}\left[\left(-\frac{1}{2 r}-\frac{\gamma+1}{8} \omega S(4 \omega r)+D_{1}\right) \sin 2 \varphi\right. \\
& \left.+\left(\frac{\gamma+1}{8} \omega[\ln r-C(4 \omega r)]+D_{2}\right) \cos 2 \varphi+\frac{\omega}{2}\right],
\end{aligned}
$$

$$
\begin{aligned}
D_{1}= & \frac{1}{1+4 \omega^{2}}\left\{-\frac{1}{\omega^{2}}-\frac{3}{2}+3 \omega^{2}+\frac{\gamma+1}{4}\right. \\
& \left.\times \omega\left[2 \omega C(4 \omega)+\left(\frac{1}{2}-2 \omega^{2}\right) S(4 \omega r)\right]\right\},
\end{aligned}
$$

$D_{2}=\frac{1}{1+4 \omega^{2}}\left\{-\frac{7 \omega}{2}-\frac{\gamma+1}{4}\right.$

$$
\left.\times \omega\left[2 \omega S(4 \omega)-\left(\frac{1}{2}-2 \omega^{2}\right) C(4 \omega)\right]\right\},
$$

$S(x)=\operatorname{si}(x) \cos x-\operatorname{ci}(x) \sin x$,

$C(x)=\operatorname{ci}(x) \cos x+\operatorname{si}(x) \sin x$.

Here, $\operatorname{si}(x)$ and $\operatorname{ci}(x)$ are, as usual, the sine integral and the cosine integral defined by

$\operatorname{si}(x)=-\int_{x}^{\infty} \frac{\sin t}{t} d t, \quad \operatorname{ci}(x)=-\int_{x}^{\infty} \frac{\cos t}{t} d t$.

\section{APPENDIX B: EXPLICIT FORMS FOR THE FUNCTIONS $a$, AND THE CONSTANTS $b$,}

The $a_{i}$ 's $(1 \leqslant i \leqslant 12)$ used in Eq. (35) are defined as follows:

$$
\begin{aligned}
a_{1}= & \frac{3 D_{2}}{\omega}-\frac{5 \gamma+23}{4}+\frac{3(\gamma+1)}{8}[\ln r-C(4 \omega r)], \\
a_{2}= & \frac{9 D_{1}}{\omega}+\frac{3(\gamma+1)}{8} S(4 \omega r), \\
a_{3}= & -\frac{3(\gamma+1) D_{2}}{\omega}-\frac{3(\gamma+1)}{8} \\
& \times[(\gamma+1) \ln r-(\gamma-3) C(4 \omega r)] \\
a_{4}= & -\frac{3 D_{1}}{\omega}+\frac{3(\gamma+1)}{8} S(4 \omega r), \\
a_{5}= & \frac{9 D_{2}}{\omega}-\frac{(2 \gamma+11)(\gamma+1)}{8}+\frac{3(\gamma+1)}{8} \\
& \times[3 \ln r+C(4 \omega r)], \\
a_{6}= & \frac{3(\gamma+1) D_{1}}{\omega}-\frac{3(\gamma+1)(\gamma-3)}{8} S(4 \omega r), \\
a_{7}= & \frac{D_{2}}{\omega}+\frac{3 \gamma+5}{4}+\frac{\gamma+1}{8}[\ln r-C(4 \omega r)]
\end{aligned}
$$




$$
\begin{aligned}
a_{8}= & \frac{D_{1}}{\omega}+\frac{3(\gamma+1)}{8} S(4 \omega r) \\
a_{9}= & \frac{(\gamma+1) D_{2}}{\omega}+\frac{\gamma+1}{8}[(\gamma+1) \ln r \\
& -(\gamma-3) C(4 \omega r)] \\
a_{10}= & -\frac{D_{1}}{\omega}+\frac{\gamma+1}{8} S(4 \omega r) \\
a_{11}= & \frac{D_{2}}{\omega}+\frac{2 \gamma^{2}+5 \gamma+11}{8} \\
& +\frac{\gamma+1}{8}[\ln r+3 C(4 \omega r)] \\
a_{12}= & -\frac{(\gamma+1) D_{1}}{\omega}+\frac{(\gamma+1)(\gamma-3)}{8} S(4 \omega r),
\end{aligned}
$$

(B12)

where constants $D_{1}$ and $D_{2}$ are defined by Eqs. (A3) and (A4) in Appendix A, respectively.

The constants $b_{i}(1 \leqslant i \leqslant 4)$ that appear in Eq. (36) are given by

$$
\begin{aligned}
b_{1}= & -\frac{9}{4 \omega}+\frac{41 \omega}{8}-\frac{2 \gamma+11}{8} \omega^{3}-\left(1-4 \omega^{2}\right) D_{2} \\
& +\left(2 \omega^{3}-3 \omega\right) D_{1}+(\gamma+1) / 8 \\
& \times\left[\omega C(4 \omega)-\left(\omega^{2}+2 \omega^{4}\right) S(4 \omega)\right], \quad(\mathrm{B} 13) \\
b_{2}= & -\frac{33}{8}+\frac{3}{4 \omega^{2}}+\frac{2 \gamma+31}{8} \omega^{2}+\left(1-4 \omega^{2}\right) D_{1} \\
& +\left(2 \omega^{3}-3 \omega\right) D_{2}-(\gamma+1) / 8 \\
& \times\left[\omega S(4 \omega)+\left(\omega^{2}+2 \omega^{4}\right) C(4 \omega)\right], \\
b_{3}= & \frac{3}{4 \omega}-\frac{3 \omega}{8}-\frac{2 \gamma+11}{8} \omega^{3}+D_{2}+\left(2 \omega^{3}+\omega\right) D_{1} \\
& -[(\gamma+1) / 8]\left[\left(\omega-4 \omega^{3}\right) C(4 \omega)\right. \\
& \left.-\left(3 \omega^{2}-2 \omega^{4}\right) S(4 \omega)\right], \\
b_{4}= & \frac{9}{8}-\frac{3}{4 \omega^{2}}-\frac{2 \gamma-5}{8} \omega^{2}-D_{1}+\left(2 \omega^{3}+\omega\right) D_{2} \\
& +[(\gamma+1) / 8]\left[\left(\omega-4 \omega^{3}\right) S(4 \omega)\right. \\
& \left.+\left(3 \omega^{2}-2 \omega^{4}\right) C(4 \omega)\right] .
\end{aligned}
$$

\section{APPENDIX C: EXPLICIT FORMS FOR THE FUNCTIONS $c$, AND THE RELATED FUNCTIONS AND CONSTANTS}

The explicit forms of the $c_{i}$ 's $(1 \leqslant i \leqslant 10)$ used in the exact solution for $\Phi_{3}$, Eq. (38), are given by

$c_{1}=-\frac{1}{10}+\frac{3 D_{2}}{2 \omega}+\frac{3(\gamma+1)}{16}[\ln r-C(4 \omega r)]$,

$c_{2}=[(\gamma+1) / 2 \omega]\left\{D_{1}[C(6 \omega r)-\ln r]+D_{2} S(6 \omega r)\right\}$

$+g_{1} S(6 \omega r)+E_{1} / \omega^{4}+\left[(\gamma+1)^{2} / 16\right]$

$\times\left[S(6 \omega r) \ln r+L_{1}(4 \omega r)+L_{7}(6 \omega r)\right.$

$\left.+L_{3}(2 \omega r) \sin 6 \omega r-L_{4}(2 \omega r) \cos 6 \omega r\right]$,

$c_{3}=37 / 80+5 \gamma / 48$,

$$
\begin{aligned}
c_{4}= & {[3(\gamma+1) / 16] S(4 \omega r)-3 D_{1} / 2 \omega, } \\
c_{5}= & \frac{\gamma+1}{2 \omega}\left\{D_{2}[C(6 \omega r)-\ln r]-D_{1} S(6 \omega r)\right\} \\
& +g_{1} C(6 \omega r)+\frac{E_{2}}{\omega^{4}}-\frac{1}{2}\left(\frac{\gamma+1}{4} \ln r\right)^{2} \\
& +\frac{(\gamma+1)^{2}}{16}\left[C(6 \omega r) \ln r+L_{2}(4 \omega r)-L_{8}(6 \omega r)\right. \\
& \left.-L_{3}(2 \omega r) \cos 6 \omega r-L_{4}(2 \omega r) \sin 6 \omega r\right], \\
c_{6}= & \frac{3}{5}+\frac{\gamma}{3}+\frac{D_{2}}{2 \omega}+\frac{\gamma+1}{16}[\ln r-C(4 \omega r)], \\
c_{7}= & -[(\gamma+1) / 2 \omega]\left\{D_{1}[C(2 \omega r)-\ln r]+D_{2} S(2 \omega r)\right\} \\
& -g_{2} S(2 \omega r)+E_{3} / \omega^{4}-\left[(\gamma+1)^{2} / 16\right] \\
& \times\left[S(2 \omega r) \ln r+L_{1}(4 \omega r)+L_{7}(2 \omega r)\right. \\
& \left.-L_{6}(2 \omega r) \cos 2 \omega r+L_{5}(2 \omega r) \sin 2 \omega r\right], \\
& -\frac{(\gamma+1)^{2}}{16}\left[C(2 \omega r) \ln r+L_{2}(4 \omega r)-L_{8}(2 \omega r)\right. \\
c_{8}= & -1 / 80-5 \gamma / 24, \\
c_{9}= & {[(\gamma+1) / 16] S(4 \omega r)-D_{1} / 2 \omega, } \\
c_{10}= & -\frac{\gamma+1}{2 \omega}\left\{D_{2}[C(2 \omega r)-\ln r]-D_{1} S(2 \omega r)\right\} \\
& \left.\left.-g_{2} C(2 \omega r)+\frac{E_{4}}{\omega^{4}}+\frac{1}{2}\left(\frac{\gamma+1}{4} \ln r\right)^{2}\right) \cos 2 \omega r-L_{6}(2 \omega r) \sin 2 \omega r\right] . \\
& (\mathrm{C} 10) \\
&
\end{aligned}
$$

Here, the $L_{i}$ 's $(1 \leqslant i \leqslant 8)$ signify the following integrals

$L_{1}(x)=-\int_{x}^{\infty} \frac{S(t)}{t} d t$

$L_{2}(x)=-\int_{x}^{\infty} \frac{C(t)}{t} d t=\frac{1}{2}\left\{[\operatorname{ci}(x)]^{2}+[\operatorname{si}(x)]^{2}\right\}$,

$L_{3}(x)=-\int_{x}^{\infty}[\operatorname{ci}(2 t) \cos t-\operatorname{si}(2 t) \sin t] \frac{d t}{t}$

$L_{4}(x)=-\int_{x}^{\infty}[\operatorname{ci}(2 t) \sin t+\operatorname{si}(2 t) \cos t] \frac{d t}{t}$,

$L_{5}(x)=-\int_{x}^{\infty}[\operatorname{ci}(2 t) \cos t+\operatorname{si}(2 t) \sin t] \frac{d t}{t}$,

$L_{6}(x)=-\int_{x}^{\infty}[\operatorname{si}(2 t) \cos t-\operatorname{ci}(2 t) \sin t] \frac{d t}{t}$,

$L_{7}(x)=-\int_{x}^{\infty} \frac{\operatorname{ci}(t)}{t} d t \sin x+\int_{x}^{\infty} \frac{\operatorname{si}(t)}{t} d t \cos x,(\mathrm{C} 17)$ $L_{8}(x)=-\int_{x}^{\infty} \frac{\operatorname{ci}(t)}{t} d t \cos x-\int_{x}^{\infty} \frac{\operatorname{si}(t)}{t} d t \sin x . \quad(\mathrm{C} 18)$ Constants $g_{1}$ and $g_{2}$ are defined by

$g_{1}=\frac{1}{40}+\frac{7}{8} \gamma+\frac{\gamma^{2}}{4}, \quad g_{2}=\frac{17}{40}+\frac{5 \gamma}{24}+\frac{\gamma^{2}}{4}$,

and the constants $E_{i}(1 \leqslant i \leqslant 4)$ are defined by Eqs. (C22)(C25) below. The constants $B, C_{1}, C_{2}, \alpha, \beta_{1}$, and $\beta_{2}$ appearing in Eq. (39) are defined as follows: 


$$
\begin{aligned}
& B=2\left(D_{1}^{2}+D_{2}^{2}\right)^{1 / 2}, \quad C_{1}=3\left(E_{1}^{2}+E_{2}^{2}\right)^{1 / 2} \omega^{-2}, \\
& C_{2}=\left(E_{3}^{2}+E_{4}^{2}\right)^{1 / 2} \omega^{-2}, \\
& \alpha=\arctan \frac{-D_{1}}{D_{2}}, \quad \beta_{1}=\arctan \frac{-E_{1}}{E_{2}}, \\
& \beta_{2}=\arctan \frac{-E_{3}}{E_{4}},
\end{aligned}
$$

where $D_{1}$ and $D_{2}$ are defined by Eqs. (A3) and (A4), respectively, and the $E_{i}$ 's $(1 \leqslant i \leqslant 4)$ are given by

$$
E_{1}=-\sin \sigma\left[P \sin (\mu+\sigma)-\Pi \Xi \sin \left(\Omega_{1}+\Psi_{1}+\sigma\right)\right],
$$

$E_{2}=\sin \sigma\left[P \cos (\mu+\sigma)-\Pi \Xi \cos \left(\Omega_{1}+\Psi_{1}+\sigma\right)\right]$,

$E_{3}=-\sin \delta\left[Q \sin (v+\delta)+\Gamma \Delta \sin \left(\Omega_{2}+\Psi_{2}+\delta\right)\right]$,

$E_{4}=\sin \delta\left[Q \cos (v+\delta)+\Gamma \Delta \cos \left(\Omega_{2}+\Psi_{2}+\delta\right)\right]$.

Here,

$$
\begin{aligned}
p_{1}= & -\frac{9}{4 \omega}+\frac{11 \omega}{2}-\left(\frac{9 \gamma}{16}+\frac{237}{80}\right) \omega^{3}+\left(\frac{13}{2} \omega^{3}-3 \omega\right) D_{1}+\left(7 \omega^{2}-1\right) D_{2} \\
& -\frac{\gamma+1}{8}\left[\left(\omega^{2}+\frac{\omega^{4}}{2}\right) S(4 \omega)-\left(\omega-3 \omega^{3}\right) C(4 \omega)\right]+\frac{(\gamma+1)^{2} \omega^{4}}{16}\left\{L_{1}(4 \omega)-3 \omega L_{2}(4 \omega)+L_{7}(6 \omega)\right. \\
& \left.-3 \omega L_{8}(6 \omega)-\cos 6 \omega\left[L_{4}(2 \omega)+3 \omega L_{3}(2 \omega)\right]+\sin 6 \omega\left[L_{3}(2 \omega)-3 \omega L_{4}(2 \omega)\right]\right\} \\
p_{2}= & -\frac{17}{4}+\frac{3}{4 \omega^{2}}+\left(\frac{9 \gamma}{16}+\frac{439}{80}\right) \omega^{2}-\left(\frac{13}{80}+\frac{7 \gamma}{8}+\frac{\gamma^{2}}{4}\right) \omega^{4}+\left(\frac{13}{2} \omega^{3}-3 \omega\right) D_{2}-\left(7 \omega^{2}-1\right) D_{1} \\
& -\frac{\gamma+1}{8}\left[\left(\omega^{2}+\frac{\omega^{4}}{2}\right) C(4 \omega)+\left(\omega-3 \omega^{3}\right) S(4 \omega)\right]+\frac{(\gamma+1)^{2} \omega^{4}}{16}\left\{L_{2}(4 \omega)+3 \omega L_{1}(4 \omega)-L_{8}(6 \omega)\right. \\
& \left.-3 \omega L_{7}(6 \omega)-\sin 6 \omega\left[L_{4}(2 \omega)+3 \omega L_{3}(2 \omega)\right]-\cos 6 \omega\left[L_{3}(2 \omega)-3 \omega L_{4}(2 \omega)\right]\right\} \\
q_{1}= & \frac{3}{4 \omega}+\left(\frac{5 \gamma}{8}-\frac{13}{80}\right) \omega^{3}+\left(\frac{5}{2} \omega^{3}+\omega\right) D_{1}+\left(\omega^{2}+1\right) D_{2}+\frac{\gamma+1}{8}\left[\left(3 \omega^{2}-\frac{\omega^{4}}{2}\right) S(4 \omega)-\left(\omega-3 \omega^{3}\right) C(4 \omega)\right] \\
& -\frac{(\gamma+1)^{2} \omega^{4}}{16}\left\{L_{1}(4 \omega)-\omega L_{2}(4 \omega)+L_{7}(2 \omega)\right. \\
& \left.-\omega L_{8}(2 \omega)-\cos 2 \omega\left[L_{6}(2 \omega)+\omega L_{5}(2 \omega)\right]+\sin 2 \omega\left[L_{5}(2 \omega)-\omega L_{6}(2 \omega)\right]\right\} \\
= & \frac{3}{4}-\frac{3}{4 \omega^{2}}-\left(\frac{7 \gamma}{8}-\frac{53}{80}\right) \omega^{2}+\left(\frac{41}{40}+\frac{13 \gamma}{24}+\frac{\gamma^{2}}{4}\right) \omega^{4}+\left(\frac{5}{2} \omega^{3}+\omega\right) D_{2}-\left(\omega^{2}+1\right) D_{1} \\
& +\frac{\gamma+1}{8}\left[\left(3 \omega^{2}-\frac{\omega^{4}}{2}\right) C(4 \omega)+\left(\omega-3 \omega^{3}\right) S(4 \omega)\right]-\frac{(\gamma+1)^{2} \omega^{4}}{16}\left\{L_{2}(4 \omega)+\omega L_{1}(4 \omega)-L_{8}(2 \omega)\right. \\
& \left.-\omega L_{7}(2 \omega)-\sin 2 \omega\left[L_{6}(2 \omega)+\omega L_{5}(2 \omega)\right]-\cos 2 \omega\left[L_{5}(2 \omega)-\omega L_{6}(2 \omega)\right]\right\}
\end{aligned}
$$

\section{APPENDIX D: A MATHEMATICAL JUSTIFICATION FOR THE DERIVATION OF A FAR-FIELD EQUATION}

The aim of this appendix is to justify the method of derivation of the far-field equation in Sec. III on the assumption that $F_{k}^{(k)}$ is an analytic function of $\varphi$. Note that this assump- tion is actually satisfied in the problems treated in Refs. 2,4 6,10 .

The solution of Eq. (1) satisfying the boundary condition (33) can be written, in implicit form, as

$$
w=\sum_{k=1}^{N} \epsilon^{k} F_{k}^{(k)}(\varphi+z w, \theta, \psi)
$$


If each $F_{k}^{(k)}$ is an analytic function of $\varphi$, then $w$ can be expanded in powers of $z$

$$
w=\sum_{n=0}^{\infty} \frac{z^{n}}{(n+1) !}\left(\frac{\partial}{\partial \varphi}\right)^{n}\left(\sum_{k=1}^{N} \epsilon^{k} F_{k}^{(k)}(\varphi, \theta, \psi)\right)^{n+1}
$$

(Lagrange's theorem). Applying the multinomial theorem to Eq. (D2) and using the relation $Z=\epsilon z / 2 \omega$ to the result gives

$$
w=\sum_{n=0}^{\infty} Z^{n} \sum_{k=1}^{N+n(N-1)} \epsilon^{k} W_{k, n},
$$

where

$$
W_{k, n}=\frac{2^{n}}{(n+1) !} \sum_{p_{i}}\left(\frac{\partial}{\partial t}\right)^{n} \prod_{i=1}^{n+1} F_{p_{i}}^{\left(p_{i}\right)} .
$$

Here, the summation in $p_{i}$ is over all sets $\left(p_{1}, p_{2}, \ldots, p_{n+1}\right)$ for which $p_{1}+p_{2}+\cdots+p_{n+1}=n+k$ and $1 \leqslant p_{1}, p_{2}, \cdots$, $p_{n+1} \leqslant N$. It then follows from Weierstrass' double series theorem that the series

$$
\sum_{n=0}^{\infty} Z^{n} W_{k, n} \quad(k=1,2, \ldots),
$$

converges and that $w$ can be expressed in a power series of $\epsilon$ as

$$
w=\sum_{k=1}^{\infty} \epsilon^{k} \sum_{n=0}^{\infty} Z^{n} W_{k, n} .
$$

A justification can be attained by confirming that one can trace back the procedure in Sec. III to formula (18) by means of Eq. (D6). Substituting Eq. (D6) into the far-field equation (1) yields

$$
\begin{aligned}
\sum_{k=1}^{\infty} \epsilon^{k} \sum_{n=0}^{\infty} Z^{n} W_{k, n}= & \frac{1}{\epsilon} \frac{\partial}{\partial t} \int_{0}^{Z}\left(\sum_{k=1}^{\infty} \epsilon^{k} \sum_{n=0}^{\infty} Z^{n} W_{k, n}\right)^{2} d Z \\
& +\sum_{k=1}^{\infty} \epsilon^{k} W_{k, 0} .
\end{aligned}
$$

Since the convergence of series (D6) has been proved, termwise integration and rearrangement of the terms in the square of sums are possible. After some calculation, one can obtain

$$
\begin{aligned}
W_{k, n-k}= & \frac{1}{n-k} \frac{\partial}{\partial t} \sum_{m=1}^{n-1} \sum_{j=1}^{k} W_{j, m-j} \\
& \times W_{k+1-j, n-m-(k+1-j)} \\
& (k=1,2, \ldots, N ; n=k+1, k+2, \ldots)
\end{aligned}
$$

[see formula (18) ]. On the other hand, it can readily be seen from Eq. (D4) that

$$
W_{k, 0}=F_{k}^{(k)} \quad(k=1,2, \ldots, N) .
$$

We here have arrived at the fact that $W_{k, n-k}$ and $F_{n}^{(k)}$ can be determined by the same formula and that they are equal for $n=k$. We therefore conclude that

$F_{n}^{(k)}=W_{k, n-k} \quad(k=1,2, \ldots, N ; n=k, k+1, \ldots)$.

A justification has thus been accomplished. Now, it is evident from Eqs. (D5) and (D10) that the right-hand side of Eq. (23) in Sec. II is a convergent series for each $k$.

${ }^{1}$ Under the condition that an acoustic Mach number $M \equiv a \omega^{*} / c=\epsilon \omega$ is sufficiently small, Inoue and Gotoh have classified the structures of sound field radiated by a uniformly pulsating sphere in an inviscid fluid into the following three types: (a) $\epsilon \ll 1$ and $\omega \gg 1$; (b) $\epsilon \ll 1$ and $\omega=O$ (1); (c) $\epsilon=\boldsymbol{O}(1)$ and $\omega \ll 1$. See Y. Inoue and Y. Gotoh, "Formation of weak shock waves caused by a sphere pulsating at large amplitude and low frequency," J. Sound Vib. 146, 269-280 (1991). In the present paper, we are concerned with the case (b).

${ }^{2}$ S. G. Kelly and A. H. Nayfeh, "Non-linear propagation of directional spherical waves," J. Sound Vib. 72, 25-37 (1980).

${ }^{3}$ S. G. Kelly and A. H. Nayfeh, "Non-linear propagation of general directional spherical waves," J. Sound Vib. 79, 145-156 (1981).

${ }^{4} \mathrm{Y}$. Inoue, S. Ishii, and T. Okigami, "Propagation of finite amplitude sound waves radiated from a pulsating sphere," J. Sound Vib. 106, $257-$ 265 (1986).

${ }^{5} \mathrm{Y}$. Inoue and T. Okigami, "Propagation of finite amplitude sound waves radiated from an oscillating rigid sphere," J. Sound Vib. 118, 199-207 (1987).

${ }^{6} \mathrm{Y}$. Inoue, T. Yano, and H. Tsujimura, "Propagation of acoustic waves and shock waves radiated by a sinusoidal pulsation of a sphere during a single period," Fluid Dyn. Res. 4, 215-227 (1988).

'J. H. Ginsberg, "Propagation of nonlinear acoustic waves induced by a vibrating cylinder. I. The two-dimensional case," J. Acoust. Soc. Am. 64, 1671-1678 (1978).

${ }^{8}$ K. A. Naugol'nykh, S. I. Soluyan, and R. V. Khokhlov, "Spherical waves of finite amplitude in a viscous thermally conducting medium," Sov. Phys. Acoust. 9, 42-46 (1963).

${ }^{9}$ D. T. Blackstock, "On plane, spherical, and cylindrical sound waves of finite amplitude in lossless fluids," J. Acoust. Soc. Am. 36, 217-219 (1964).

${ }^{10} \mathrm{~T}$. Yano and Y. Inoue, "Second order solution for non-linear sound waves radiated from an oscillating rigid sphere," J. Sound Vib. 135, 385-397 (1989).

"J. C. Lockwood, T. G. Muir, and D. T. Blackstock, "Directive harmonic generation in the radiation field of a circular piston," J. Acoust. Soc. Am. 53, 1148-1153 (1973).

${ }^{12}$ H. W. Marsh, R. H. Mellen, and W. L. Konrad, "Anomalous absorption of pressure waves from explosions in sea water," J. Acoust. Soc. Am. 38, 326-338 (1965).

${ }^{13} \mathrm{H}$. S. Heaps, "Waveform of finite amplitude derived from equations of hydrodynamics," J. Acoust. Soc. Am. 34, 355-356 (1962).

${ }^{14}$ K. A. Naugol'nykh, S. I. Soluyan, and R. V. Khokhlov, "Cylindrical waves of finite amplitude in a dissipative medium," Vestnik, Moscow State Univ., Phys. Astron., Ser. 34, 65-71 (1962).

${ }^{15}$ For the D'Alembertian operator, the symbol " $\square$ "' is often used as well as “回”,

${ }^{16}$ In Ref. 7, Ginsberg introduced an arbitrary function of the polar angle $\theta$ only, $F(\theta)$, in $\Phi_{2}$ so that he could renormalize the tangential velocity $u_{\theta}$. It is however evident that $\Phi_{2}$ cannot contain such a function $F(\theta)$. We here note that, for spherical waves, the tangential velocity $u_{\theta}$ cannot be renormalized by the strained coordinate which renormalize the radial velocity $u_{r}$ and the pressure $p$ (see Refs. 5 and 10) 